\title{
On HARQ Schemes in Satellite-Terrestrial Transmissions
}

\author{
Gaofeng Pan, SeniorMember, IEEE, Jia Ye, Student Member, IEEE, Yu Tian, and \\ Mohamed-Slim Alouini, Fellow, IEEE
}

\begin{abstract}
As an efficient supplement for terrestrial communications, e.g., fifth-generation communication networks, satellite communication has been proved as a promising choice to realize seamless coverage, due to its inherent superiority over common wireless communication systems, like large-scale coverage area and flexibility of deployment. In this paper, a satellite-terrestrial transmission (STT) system including a satellite transmitter (S) and a group of terrestrial receivers (D) is focused on. Considering the randomness of $\mathrm{D}$ and employing stochastic geometry, the outage performance and diversity gain of three hybrid automatic repeat request (HARQ) schemes, generalized slotted ALOHA (GSA), repetition time diversity (RTD) and general incremental redundancy (IR), are respectively studied. In detail, the exact closedform analytical expression for the outage probability (OP) is derived for the GSA scheme, while the approximated expression for the OP of RTD scheme is presented and a useful expression for the OP of IR scheme is derived. Furthermore, the diversity gains of the three considered HARQ schemes are respectively investigated in STT scenarios. Finally, some numerical results are provided to validate the proposed analysis models.
\end{abstract}

\section{Index Terms}

Diversity order, hybrid automatic repeat request, outage probability, satellite-terrestrial communication, stochastic geometry.

Manuscript received Mar. 16, 2020; revised June 06, 2020; accepted Aug. 18, 2020. The associate editor coordinating the review of this paper and approving it for publication was M. Payaró. (Corresponding author: Gaofeng Pan.)

G. Pan is with Computer, Electrical and Mathematical Sciences and Engineering Division, King Abdullah University of Science and Technology (KAUST), Thuwal 23955-6900, Saudi Arabia, and he is also with the School of Information and Electronics Engineering, Beijing Institute of Technology, Beijing 100081, China.

J. Ye, Y. Tian, and M.-S. Alouni are with Computer, Electrical and Mathematical Sciences and Engineering Division, King Abdullah University of Science and Technology (KAUST), Thuwal 23955-6900, Saudi Arabia. 


\section{INTRODUCTION}

Profiting from its natural merits, e.g., multicast/broadcast functionality, large-scale coverage, long communication distance, the flexibility of deployment, and little dependence on ground-based facilities, satellite communication has already been considered and proved as a promising supplement for common terrestrial wireless communications, such as fifth-generation wireless communication systems, to realize seamless coverage and full connectivity in under-served and remote areas [1]-[5].

On the other hand, wireless channels are time-varying and error-prone, because of fading, additive noise, and interference, causing the strength of the received signal at the receiver to vary and further resulting in loss of data packets. To improve the reliability of data transmissions, hybrid automatic repeat request (HARQ) schemes have been developed to compensate for link adaptation errors, which is a combination of forward error correction and automatic repeat request (ARQ) [6], [7].

Referring to satellite-terrestrial transmission (STT) systems, the channel conditions between the satellite and the terrestrial receivers are harsher compared with traditional wireless communication systems, due to its inherent characteristics, such as the long-distance transmissions and high-speed mobility of the satellites in a highly elliptical orbit, medium earth orbit and low earth orbit (LEO). Therefore, how to ensure reliable data transmissions and seamless coverage over such challenging STT channels is still an interesting problem that merits serious investigations.

Therefore, benefiting from their inherent advantages, HARQ schemes are an indispensable and effective tool for STT systems to overcome the aforementioned problems. Then, in the following, we will present a review of the existing works on HARQ schemes according to the fading types in the considered scenarios.

Some researchers have studied the performance of HARQ schemes in Gaussian channels. For example, the authors of [8] analyzed the performance of a hybrid selective repeat/multi-copy ARQ scheme to transmit fragmented internet protocol packets in terms of bit error rate, internet protocol packet size, and fragmentation size, and the throughput performance of truncated ARQ schemes was examined and optimized in Gaussian block fading channels [9].

There are some more works presented to investigate the performance of HARQ schemes over Rayleigh fading channel, which is a popular small-scale fading channel without line-of-sight (LOS) components. The average throughput and outage probability (OP) of a multi-relay delay-limited HARQ system were analyzed in [10], in which an opportunistic relaying scheme and decode-and-forward relaying scheme are considered. The closed-form expressions were derived for the OP, delay limited throughput, and mean transmission time of the HARQ scheme, while considering time-correlated interference and feedback 
errors [11]. Analytical expressions were derived for the OP, the average number of transmissions (ANT), and the average transmission rate (ATR) of HARQ-incremental redundancy (IR) and HARQ with code combining over a relay channel [12]. In [13], a closed-form expression for the effective capacity of the HARQ-IR scheme was proposed for small values of the quality-of-service exponent. The authors of [14] derived general analytical expressions for the optimal throughput and optimal rate point in a matrix exponential distribution form. Analytical expressions were derived for the $\epsilon$-outage capacity, ATN, and ATR of HARQ-IR over double Rayleigh fading channels in [15], while assuming a maximum number of HARQ rounds.

Considering the high probability of the occurrence of time correlation in practical fading scenarios, some researchers paid their attention to the performance of HARQ schemes over time-correlated fading channels. Considering time-correlated Rayleigh fading channels, the OP, ATN, long term average throughput, and energy efficiency of HARQ-IR were analyzed in [16], [17]. Asymptotic outage performance and optimal rate selection were studied for HARQ schemes over time-correlated Nakagami- $m$ fading channels in [18] and [19], respectively. Moreover, the performance of HARQ schemes in free-space optical channels was investigated in [20], [21]. Moreover, by considering Nakagami- $m$ fading, outage performance of HARQ-IR in a mobile ad hoc network was analyzed [22], and the secure transmission in HARQ-assisted non-orthogonal multiple access networks was studied [23].

So far, there are a few works presented to deal with the HARQ schemes applied in STT systems. A HARQ scheme was proposed in [24] to provide both high system throughput and high system reliability over satellite channels. In [25], two HARQ schemes, termed hybrid go-back-N and hybrid selective-repeat, were proposed for point-to-multi-point communications over broadcast channels, such as satellite broadcast links. In [26], a satellite multiple access protocol was proposed and investigated, which combines the characteristics of the spread slotted-ALOHA protocol, code division multiple access, and the HARQ error control and re-transmission scheme. The authors of [27] studied the performance of transmission control protocol enhancements for a hybrid terrestrial-satellite network, and the expressions for the end-to-end throughput with link-layer suitable for reliable-ARQ and transmission control protocol splitting were obtained. The closed-form expressions for the mean number of transmission rounds were derived by using Laplace transforms for reliable data delivery in ARQ and HARQ schemes in multipleinput multiple-output STT networks [28].

Obviously, there exist some inherent challenges for implementing the HARQ schemes in STT scenarios, e.g., the path-loss arisen from the long-distance transmission, the small-scale fading attributed to the complex surrounding environments of the terrestrial receivers, and the random distribution of the terrestrial 
receivers, etc. Therefore, though there exist some works reported to study the HARQ schemes adopted either in common wireless systems or in satellite communication systems, it is obvious that there are still some blanks in the research area of the HARQ schemes in STT systems. For instance, how does the channel between the satellite and the terrestrial receivers affect the performance of HARQ schemes? And what is the impact of the randomness of the terrestrial receiver's position on the performance of HARQ scheme?

To answer these aforementioned questions and fully understand the performance of HARQ schemes in STT scenarios, in this paper we consider an STT system consisting of a satellite (S) and a group of terrestrial receivers. $\mathrm{S}$ delivers information massages to these terrestrial receivers via multicast transmissions, while the terrestrial receivers are uniformly distributed in the coverage area of S. Three HARQ schemes, generalized slotted ALOHA (GSA), repetition time diversity (RTD) and general IR, are respectively considered at $\mathrm{S}$ to implement the required re-transmissions, to realize the reliable information delivery between $\mathrm{S}$ and all terrestrial receivers. Because transmission errors can not be avoided and ignored in such long-distance STT scenarios.

Differing from the existing works on the HARQ schemes for STT [24]-[28], analysis models are set up to study and understand the performance of three classic HARQ schemes, namely, GSA, RTD, and general IR schemes in satellite-to-terrestrial fading channels while considering the randomness of the positions of terrestrial receivers. The best of our knowledge, this work is the first to build up such performance analysis models for the HARQ schemes for STT, which can also serve as a useful reference for investigating the performance of other HARQ scheme for STT. The main contributions of this work are summarized as follows:

1) The exact closed-form analytical expression is presented for the OP of the GSA scheme;

2) The approximated analytical expression for the OP of RTD scheme is derived, while a useful expression for the OP of HARQ-IR scheme is presented;

3) The diversity order is derived for the three considered HARQ schemes.

The rest of this paper is organized as follows. In Section II, the considered STT system is described. In Section III and IV, the outage and diversity analyses are conducted for the three considered HARQ schemes, respectively. In Section V, numerical results are presented and discussed. Finally, the paper is concluded with some remarks in Section VI. 


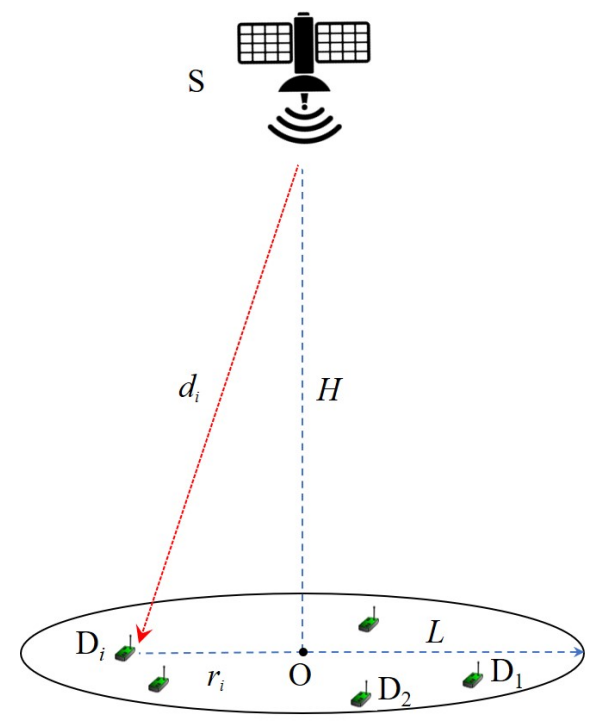

Fig. 1: System model.

\section{SYSTEM MODEL}

In this work, an STT system, which consists of a satellite transmitter (S), and a group of terrestrial receivers $\left(\mathrm{D}_{i}, 1 \leq i \leq N\right)$, is considered, as presented in Fig. 1. It is assumed that $\mathrm{S}$ and all terrestrial receivers are equipped with a single transmit and a single receiving antenna.

\section{A. The Distribution Model of Terrestrial Receivers}

For tractability purposes, in this work we also treat the coverage area of the beam of $\mathrm{S}$ as a circle, $\mathcal{S}$, with radius $L$, where the projection of $S$ is located at the centre of the circle. The location of terrestrial receivers is modeled as a homogeneous Poisson Point process (PPP) with density $\lambda$. The number of terrestrial receivers, which denoted by $N(N \geq 1)$, is Poisson distributed i.e., $P\{N=k\}=\left(\mu_{\mathcal{S}}^{k} / k !\right) \exp \left(-\mu_{\mathcal{S}}\right)$, where $\mu_{\mathcal{S}}=\pi L^{2} \lambda$ is the mean measure. Then, the $N(N \geq 1)$ terrestrial receivers can be modeled as a set of independent and identically distributed points in the circle, $\mathcal{S}$, denoted by $\mathcal{W}$. Therefore, the distance between terrestrial receivers and the projection of $\mathrm{S}(O)$ can be calculated from $\mathcal{W}$, the probability density function (PDF) of which can be given by [29]-[32]

$$
f_{\mathcal{W}}(w)=\frac{\lambda}{\mu(\mathcal{S})}=\frac{1}{\pi L^{2}} .
$$


So, the cumulative distribution function (CDF) of $r_{i}(1 \leq i \leq N)$ can be derived as

$$
F_{r_{i}}(x)=\int_{\mathcal{S}} f_{\mathcal{W}}(w) d w=\int_{0}^{2 \pi} \int_{0}^{x} \frac{1}{\pi L^{2}} r d r d \theta=\frac{x^{2}}{L^{2}} .
$$

Therefore, the PDF $r_{i}(1 \leq i \leq N)$ can be obtained as

$$
f_{r_{i}}(x)=\frac{2 x}{L^{2}} .
$$

Further, we can derive the PDF of $d_{i}=\sqrt{H_{1}^{2}+r_{i}^{2}}(1 \leq i \leq N)$ as

$$
f_{d_{i}}(x)=\frac{2 x}{L^{2}},
$$

where $H_{1} \leq x \leq \sqrt{H_{1}^{2}+L^{2}}$.

\section{B. HARQ Schemes}

In this work, $\mathrm{S}$ is designed to deliver information messages to terrestrial receivers $\left(\mathrm{D}_{i}, 1 \leq i \leq N\right)$ by using a multicast transmission. The time axis is divided into slots with duration $T$ seconds, and all terrestrial receivers are assumed to be synchronized so they are with a common time reference.

Under the ARQ protocol, the information messages at $\mathrm{S}$, the length of which is $b$ bits, are encoded into $K$ subblocks, which are of the length of $L$. In other words, $\mathrm{S}$ encodes its information message of $b$ bits each into a channel code $c$ of length $L K$ over the complex numbers chosen from the codebook, $\mathcal{C} \in \mathbb{C}^{L K}$. This codeword can be divided into $K$ subblocks of length $L$. For each subblock $\boldsymbol{c}_{j} \in\left\{\boldsymbol{c}_{1}, \boldsymbol{c}_{2}, \cdots, \boldsymbol{c}_{K}\right\}$, all information for successfully decoding the original information message is included. Consequently, at each receiver, the original message can be rebuilt by using each subblock or any combination of these subblocks, while the receiving SNR is large enough.

At the start of the $j$ th $(1 \leq j \leq K)$ time slot, $\mathrm{S}$ encapsulates the subblock $\boldsymbol{c}_{j}$ into one packet, which takes slightly less than $T$ seconds to allow $\mathrm{S}$ to process the feedback information from the terrestrial receivers, and transmits the packet to all terrestrial receivers for the $j$ th $(1 \leq j \leq K)$ round of multicasting the $b$ bits of information message. In each round of delivering $b$ bits of information message, if a terrestrial user is unable to decode the message, it sends a negative acknowledgment (NACK) message through an error-free and zero-delay feedback channel to S. If the decoding is successful, the terrestrial receiver remains silent and does not feedback any messages. This NACK message is received by $\mathrm{S}$ at the end of each time slot. Then, when at least one NACK message is obtained at S, S will deliver another packet containing $\boldsymbol{c}_{j+1}$ to all terrestrial receivers. If $\mathrm{S}$ does not receive a NACK message via the feedback channel, it is regarded that the transmission of the bits information message is complete, and then S starts to deliver 
another $b$ bits information message again following the aforementioned process. Furthermore, if at least one NLCK message is overheard by $\mathrm{S}$ at the end of the $K$ time slot, the transmitted message will be discarded by $\mathrm{S}$ and an outage event occurs for the whole system.

In the $j$ th $(1 \leq j \leq K)$ time slot, upon receiving the packet containing $\boldsymbol{c}_{j}$, the terrestrial receiver will

decode the transmitted message by using the received signals from the subblocks $\left\{\boldsymbol{c}_{(j-M+1)^{+}}, \cdots, \boldsymbol{c}_{j}\right\}$, where $M$ is the memory size at the terrestrial receiver, and $(j-M+1)^{+}=\max \{1, j-M+1\}$. Namely, the $M$ most recently received packets are adopted to decode the original transmitted message at each terrestrial receiver. Moreover, generally, if a terrestrial receiver successfully decodes the transmitted message, it will stay silent in the next time slot.

\section{Signal Model}

In this work, it is assumed that all channels experience independent and identically distributed (i.i.d.) block-fading. That is to say, the channel gain for each channel remains constant over a block/slot but varies independently from one block/slot to another [33]. The received signal at the $i$ th $(1 \leq i \leq N)$ terrestrial receiver, $\mathrm{D}_{i}$, in the $j$ th $(1 \leq j \leq K)$ time slot is given by

$$
y_{\mathrm{D}_{i}}[j]=\sqrt{P_{\mathrm{S}} d_{i}^{-n}} h_{\mathrm{SD}_{i}}[j] x[j]+n_{\mathrm{D}_{i}},
$$

where $P_{\mathrm{S}}$ is the transmit power at $\mathrm{S}, d_{i}$ is the distance between $\mathrm{S}$ and $\mathrm{D}_{i}, n$ is the path-loss factor over $\mathrm{S}-\mathrm{D}_{i}$ link, $h_{\mathrm{SD}_{i}}[j]$ is the channel gain for $\mathrm{S}-\mathrm{D}_{i}$ link in the $j$ th $(1 \leq j \leq K)$ time slot, $x[j]$ is the transmitted subblock in the $j$ th $(1 \leq j \leq K)$ time slot, and $n_{\mathrm{D}_{i}}$ denotes the complex additive white Gaussian noise (AWGN) at $\mathrm{D}_{i}$.

Furthermore, in this work Shadowed-Rician (SR) model is adopted to describe the statistical distribution of the STT link from $\mathrm{S}$ to $\mathrm{D}_{i}$, which has been proved to be an accurate, practical and applicable tool to evaluate performance for satellite propagation environments in various frequency bands, e.g., the UHFband, L-band, S-band, Ku-band, and Ka-band.

Without loss of generality, the PDF of the power gain, $\left|h_{\mathrm{SD}_{i}}[j]\right|^{2}$, for $\mathrm{S}-\mathrm{D}_{i}$ link in $\mathrm{SR}$ channel is given as [34]

$$
f_{\left|h_{\mathrm{SD}_{i}}[j]\right|^{2}}(x)=\alpha \exp (-\beta x){ }_{1} F_{1}(m ; 1 ; \delta x), \quad x \geq 0,
$$

where $\alpha=\left(\frac{2 b m}{2 b m+\Omega}\right)^{m} /(2 b), \beta=\frac{1}{2 b}$ and $\delta=\frac{\Omega}{2 b(2 b m+\Omega)}, \Omega$ and $2 b$ are the average power of the LOS and multipath components, respectively. $m$ is the fading severity parameter (When $m=0$, it denotes the case without LOS; when $0<m<\infty$, it stands for the case of both small-scale fading and LOS; when 
$m=\infty$, it represents the $\operatorname{LOS}$ case), and ${ }_{1} F_{1}(\cdot ; \cdot ; \cdot)$ is the confluent hypergeometric function of first kind [35].

Then, it is easy to obtain the received signal-to-noise ratio (SNR) at $\mathrm{D}_{i}$ in the $j$ th $(1 \leq j \leq K)$ time slot as

$$
\gamma_{i j}=\frac{P_{\mathrm{S}}}{\sigma^{2}} \frac{\left|h_{\mathrm{SD}_{i}}[j]\right|^{2}}{d_{i}^{n}}=\frac{\lambda_{j}}{d_{i}^{n}},
$$

where $\lambda_{j}=P_{\mathrm{S}}\left|h_{\mathrm{SD}_{i}}[j]\right|^{2} / \sigma^{2}=\bar{\lambda}\left|h_{\mathrm{SD}_{i}}[j]\right|^{2}, \bar{\lambda}=P_{\mathrm{S}} / \sigma^{2}$ is the transmit SNR, and $\sigma^{2}$ is the average power of the AWGN at $\mathrm{D}_{i}$.

By variable substitution and applying Kummers transform, the PDF and CDF of $\lambda_{j}$ can be presented as

$$
f_{\lambda_{j}}(x)=\alpha \sum_{k=0}^{m-1} \frac{\varsigma(k)}{\bar{\lambda}^{k+1}} x^{k} \exp \left(-\frac{\beta-\delta}{\bar{\lambda}} x\right)
$$

and

$$
F_{\lambda_{j}}(x)=1-\alpha \sum_{k=0}^{m-1} \frac{\varsigma(k)}{\bar{\lambda}^{k+1}} \sum_{p=0}^{k} \frac{k !}{p !}\left(\frac{\beta-\delta}{\bar{\lambda}}\right)^{-(k+1-p)} x^{p} \exp \left(-\frac{\beta-\delta}{\bar{\lambda}} x\right)
$$

respectively, where $\varsigma(k)=\frac{(-1)^{k}(1-m)_{k} \delta^{k}}{(k !)^{2}}$ and $(t)_{k}=t(t+1) \cdots(t+k-1)$ is the Pochhammer symbol [35].

Then, according to the ARQ protocol presented in the previous subsection and adopting maximum ratio combining scheme at each receiver to process the multiple received signals, the accumulated mutual information at $\mathrm{D}_{i}$ used for decoding in the $j$ th $(1 \leq j \leq K)$ time slot is then given by

$$
\begin{aligned}
I_{i j} & =\sum_{s=(j-M+1)^{+}}^{j} \log _{2}\left(1+\gamma_{i s}\right) \\
& =\sum_{s=(j-M+1)^{+}}^{j} \log _{2}\left(1+\frac{\lambda_{s}}{d_{i}^{n}}\right) .
\end{aligned}
$$

\section{Performance Metric}

In this work, we mainly concern the impacts of the adopted HARQ schemes on the performance of the considered STT system. Specifically, the outage performance and diversity gain will be studied in the following parts of this paper, which are respectively defined as

1) Outage probability: The probability that an originally transmitted message cannot be successfully decoded by at least one terrestrial receiver after $K$ rounds of deliveries. 
2) Diversity gain: Multiple copies of the transmitted signal can be exploited during the decoding process.

To facilitate the following analysis, some assumptions are made as follows:

1) Infinite-size buffer is available at $S$, which means that there always exist information messages at $\mathrm{S}$ to be delivered to terrestrial receivers;

2) The processing delay at $\mathrm{S}$ and terrestrial receivers and transmission delays are omitted.

\section{Outage AnAlysis}

In this section, outage analysis will be carried out for three hybrid-ARQ schemes: GSA, RTD, and IR schemes, respectively.

In each round of the transmission of the information message, the outage event occurs at $\mathrm{D}_{i}$ when the accumulated mutual information is less than the transmission rate, $R$, resulting in the failure of the decoding. So, the OP at $\mathrm{D}_{i}$ in the $j$ th $(1 \leq j \leq K)$ time slot can be represented as

$$
P_{\text {out }, i j}=\operatorname{Pr}\{\text { decoding error }\}=\operatorname{Pr}\left\{I_{i j}<R\right\} .
$$

Theorem 1. As there are $N(N \geq 1)$ terrestrial receivers within the coverage area of $S$ in each system realization, and all links between $S$ and the terrestrial receivers experience i.i.d. fading, the $O P$ for the whole system can be presented as

$$
P_{\text {out }}=1-\left(1-\rho^{K}\right)^{N}
$$

where $\rho=P_{\text {out }, i j}$.

Proof: For terrestrial receiver $\mathrm{D}_{i}$, the probability that it can successfully decode the transmitted information message in the $j$ th $(1 \leq j \leq K)$ time slot is written as

$$
P_{\mathrm{su}, i j}=P_{\mathrm{out}, i j}{ }^{j-1}\left(1-P_{\mathrm{out}, i j}\right) .
$$

So, the probability that $\mathrm{D}_{i}$ can successfully decode the transmitted information message during the $K$ rounds of the transmissions can be given as

$$
P_{\mathrm{su}, i}=\sum_{j=1}^{K} P_{\mathrm{su}, i j}=\sum_{j=1}^{K} P_{\mathrm{out}, i j}{ }^{j-1}\left(1-P_{\mathrm{out}, i j}\right) .
$$

Considering that all links between $S$ and the terrestrial receivers experience i.i.d. fading, we have $P_{\text {out }, i j}=P_{\text {out }, p q}$ for $\{(i, j, p, q) \mid\{i, j, p, q\} \in\{1,2, \cdots, N\}\}$. Then, the OP for the whole system can be 
derived as

$$
\begin{aligned}
P_{\mathrm{out}} & =1-\prod_{i=1}^{N} P_{\mathrm{su}, i} \\
& =1-\prod_{i=1}^{N} \sum_{j=1}^{K} P_{\mathrm{out}, i j}{ }^{j-1}\left(1-P_{\mathrm{out}, i j}\right) \\
& =1-\prod_{i=1}^{N}\left(1-\rho^{K}\right) \\
& =1-\left(1-\rho^{K}\right)^{N},
\end{aligned}
$$

where in this work we let $\rho=P_{\mathrm{out}, \text { ij }}$ for simplification.

Then, the proof is completed.

\section{A. GSA Scheme}

Under the GSA scheme, the memory size at the terrestrial receivers $M=1$. In other words, all terrestrial receivers only adopt the latest received signal to decode the transmitted message, i.e., in the $j$ th $(1 \leq j \leq K)$ time slot, the received signal of the packet is only used for decoding.

Therefore, the accumulated mutual information at $\mathrm{D}_{i}$ in the $j$ th $(1 \leq j \leq K)$ time slot is

$$
I_{i j}=\log _{2}\left(1+\gamma_{i j}\right)=\log _{2}\left(1+\frac{\lambda_{j}}{d_{i}^{n}}\right) .
$$

Accordingly, the OP under GSA scheme in the $j$ th $(1 \leq j \leq K)$ time slot can be written as

$$
\begin{aligned}
P_{\text {out }, i j} & =\operatorname{Pr}\left\{\log _{2}\left(1+\frac{\lambda_{j}}{d_{i}^{n}}\right)<R\right\} \\
& =\operatorname{Pr}\left\{\frac{\lambda_{j}}{d_{i}^{n}}<\varepsilon\right\},
\end{aligned}
$$

where $\varepsilon=2^{R}-1$.

Theorem 2. Considering the randomness of the channel gain between $S$ and $\mathrm{D}_{i}$, and the randomness of the position of $\mathrm{D}_{i}$, the OP under GSA scheme in the $j$ th $(1 \leq j \leq K)$ time slot can be derived as

$$
P_{\text {out }, i j}=1-\frac{2 \alpha}{n L^{2}} \sum_{k=0}^{m-1} \frac{\varsigma(k)}{\bar{\lambda}^{k+1}} \sum_{p=0}^{k} \frac{k !}{p !}\left(\frac{\beta-\delta}{\bar{\lambda}}\right)^{-\left(k+\frac{2}{n}+1\right)} \varepsilon^{-\frac{2}{n}}\left[\gamma\left(p+\frac{2}{n}, y_{\max }\right)-\gamma\left(p+\frac{2}{n}, y_{\min }\right)\right],
$$

where $y_{\min }=\frac{\varepsilon(\beta-\delta)}{\bar{\lambda}} H_{1}^{n}, y_{\max }=\frac{\varepsilon(\beta-\delta)}{\bar{\lambda}}\left(H_{1}^{2}+L^{2}\right)^{\frac{n}{2}}$, and $\gamma(a, x)=\int_{0}^{x} t^{a-1} \exp (-t) d t$ is the lower incomplete gamma function. 
Proof: Please refer to Appendix I.

Therefore, the OP for the whole system under the GSA scheme can be obtained by substituting (18) into (12).

\section{B. RTD Scheme}

Under the RTD scheme, the information messages are encoded by using repetition coding, leading to the fact that all the packets transmitted in different time slots of the $K$ rounds are the same. So, under RTD scheme, the accumulated mutual information at $\mathrm{D}_{i}$ used for decoding in the $j$ th $(1 \leq j \leq K)$ time slot is given by

$$
\begin{aligned}
I_{i j} & =\log _{2}\left(1+\sum_{s=(j-M+1)^{+}}^{j} \gamma_{i s}\right) \\
& =\log _{2}\left(1+\sum_{s=(j-M+1)^{+}}^{j} \frac{\lambda_{s}}{d_{i}^{n}}\right) \\
& =\log _{2}\left(1+\frac{1}{d_{i}^{n}} \sum_{s=(j-M+1)^{+}}^{j} \lambda_{s}\right) .
\end{aligned}
$$

Thus, the OP under RTD scheme in the $j$ th $(1 \leq j \leq K)$ time slot can be written as

$$
\begin{aligned}
P_{\text {out }, i j} & =\operatorname{Pr}\left\{\log _{2}\left(1+\frac{1}{d_{i}^{n}} \sum_{s=(j-M+1)^{+}}^{j} \lambda_{s}<R\right\}\right. \\
& =\operatorname{Pr}\left\{\frac{1}{d_{i}^{n}} \sum_{s=(j-M+1)^{+}}^{j} \lambda_{s}<\varepsilon\right\} .
\end{aligned}
$$

Theorem 3. Considering the randomness of the channel gain between $S$ and $\mathrm{D}_{i}$, and the randomness of the position of $\mathrm{D}_{i}$, the OP under RTD scheme in the $j$ th $(1 \leq j \leq K)$ time slot can be derived as

$$
P_{\text {out }, i j}=\alpha^{\mathcal{M}} \sum_{l=0}^{c}\left(\begin{array}{l}
c \\
l
\end{array}\right) \beta^{c-l}[H(l, d)+\nu \delta H(l, d+1)],
$$

where $\mathcal{M}=j-[j-M]^{+}, c=[d-\mathcal{M}]^{+}, \nu=m \mathcal{M}-d, d=\max \{\mathcal{M},\lfloor m \mathcal{M}\rfloor\},\lfloor x\rfloor$ denotes the largest integer not larger than $x,[x]^{+}$indicates that if $x \leq 0$, then use $x=0, t_{\max }=\left(H_{1}^{2}+L^{2}\right)^{\frac{n}{2}}$, 


$$
\begin{aligned}
& t_{\min }=H_{1}{ }^{n}, J=\frac{\varepsilon(\beta-\delta)}{\lambda}, \frac{2(\beta-\delta)^{\frac{l-d-1}{2}}}{n L^{2} \Gamma(d)}\left(\frac{\varepsilon}{\bar{\lambda}}\right)^{\frac{d-l-1}{2}}\left\{t_{\max }^{\frac{d-l-1}{2}+\frac{2}{n}} G_{2,3}^{1,2}\left[J t_{\max } \mid \begin{array}{r}
\frac{3-d-l}{2}, \frac{3-d+l}{2}-\frac{2}{n} \\
\frac{d-l+1}{2}, \frac{1-d+l}{2}-\frac{2}{n}, \frac{1-d+l}{2}
\end{array}\right] \begin{array}{l}
\frac{d-l-1}{2}+\frac{2}{n} \\
H(l, d)=t_{\min }
\end{array}\right. \\
&\left.\cdot G_{2,3}^{1,2}\left[J t_{\min } \mid \begin{array}{l}
\frac{3-d-l}{2}, \frac{3-d+l}{2}-\frac{2}{n} \\
\frac{d-l+1}{2}, \frac{1-d+l}{2}-\frac{2}{n}, \frac{1-d+l}{2}
\end{array}\right]\right\}
\end{aligned}
$$

and $G_{p, q}^{m, n}\left[x \mid \begin{array}{l}a_{1}, \cdots, a_{p} \\ b_{1}, \cdots, b_{q}\end{array}\right]$ is the Meijer's G-function, as defined by [35, Eq. (9.301)].

Proof: Please refer to Appendix II.

Then, the OP for the whole system under the RTD scheme can be obtained by substituting (21) into (12).

\section{HARQ-IR Scheme}

Under the HARQ-IR scheme, a general IR code at $\mathrm{S}$ is adopted, which indicates that the transmitted packets in different timeslots of the $K$ rounds of the transmission will be different from each other. At the receiver, in the $j$ th $(1 \leq j \leq K)$ time slot, the signal of the previous $j-(j-M+1)^{+}$received packets are adopted to decode the transmitted information message. So, under this case, the accumulated mutual information at $\mathrm{D}_{i}$ used for decoding in the $j$ th $(1 \leq j \leq K)$ time slot can be given by (10).

Accordingly, the OP under HARQ-IR scheme is then given by

$$
\begin{aligned}
& P_{\text {out }, i j}=\operatorname{Pr}\left\{\sum_{s=(j-M+1)^{+}}^{j} \log _{2}\left(1+\gamma_{i s}\right)<R\right\} \\
& =\operatorname{Pr}\left\{\log _{2} \prod_{s=(j-M+1)^{+}}^{j}\left(1+\gamma_{i s}\right)<R\right\} \\
& =\operatorname{Pr}\left\{\prod_{s=(j-M+1)^{+}}^{j}\left(1+\gamma_{i s}\right)<2^{R}\right\} \text {. }
\end{aligned}
$$

Observing the above equation, one can see that it is impossible to derive the exact closed-form analytical expression for the CDF of $\prod_{s=(j-M+1)^{+}}^{j}\left(1+\frac{\lambda_{s}}{d_{i}^{n}}\right)$, while considering the complexity of the statistical characteristics of $\lambda_{s}$ shown by (8) and (9), as well as the complexity of $\prod_{s=(j-M+1)^{+}}^{j}\left(1+\frac{\lambda_{s}}{d_{i}^{n}}\right)$. In the following, we will investigate the OP in high SNR region for the considered system. Then, using $1+\gamma_{i s} \approx$ 
$\gamma_{i s},(23)$ can be further rewritten as

$$
\begin{aligned}
P_{\text {out }, i j} & =\operatorname{Pr}\left\{\prod_{s=(j-M+1)^{+}}^{j}\left(1+\bar{\lambda} \frac{\left|h_{\mathrm{SD}_{i}}[s]\right|^{2}}{d_{i}^{n}}\right)<2^{R}\right\} \\
& \approx \operatorname{Pr}\left\{\prod_{s=(j-M+1)^{+}} \bar{\lambda} \frac{\left|h_{\mathrm{SD}_{i}}[s]\right|^{2}}{d_{i}^{n}}<2^{R}\right\} \\
& =\operatorname{Pr}\left\{\frac{\bar{\lambda}^{\mathcal{M}}}{d_{i}^{n \mathcal{M}}} \prod_{s=(j-M+1)^{+}}^{j}\left|h_{\mathrm{SD}_{i}}[s]\right|^{2}<2^{R}\right\} \\
& =\operatorname{Pr}\left\{\frac{Y}{Z}<2^{R}\right\},
\end{aligned}
$$

where $Y=\prod_{s=(j-M+1)^{+}}^{j}\left|h_{\mathrm{SD}_{i}}[s]\right|^{2}, Z=\frac{d_{i}^{n \mathcal{M}}}{\lambda^{\mathcal{M}}}$, and $z_{\min }=\frac{H_{1}^{n \mathcal{M}}}{\bar{\lambda}^{\mathcal{M}}} \leq Z \leq z_{\max }=\frac{\left(H_{1}{ }^{2}+L^{2}\right)^{\frac{n \mathcal{M}}{2}}}{\bar{\lambda}^{\mathcal{M}}}$.

Theorem 4. Considering that all links between $S$ and the terrestrial receivers suffer i.i.d. SR fading, the $C D F$ of $W=Y / Z$ (or equivalently the OP under HARQ-IR scheme) can be derived as

$$
\begin{aligned}
P_{\text {out }, i j}= & F_{W}\left(2^{R}\right)=\frac{1}{2}-\frac{1}{\pi} \\
& \cdot \int_{0}^{\infty} \frac{\operatorname{Im}\left\{2^{-\jmath R t} \jmath \frac{2 \lambda^{\frac{2}{n}}}{L^{2}} \frac{z_{\max }^{\frac{2}{n}-\jmath t}-z_{\min }^{\frac{2}{\operatorname{MM}}-\jmath t}}{2 \jmath+n \mathcal{M} t}\left[\alpha \beta^{-(\jmath t+1)} \Gamma(\jmath t+1) \cdot{ }_{2} F_{1}\left(m, \jmath t+1 ; 1 ; \frac{\delta}{\beta}\right)\right]^{\mathcal{M}}\right\}}{t} d t
\end{aligned}
$$

where $\mathrm{J}=\sqrt{-1}$ is the imaginary unit, $\operatorname{Im}\{\cdot\}$ represents the imaginary part of a complex number, and ${ }_{2} F_{1}(\cdot, \cdot ; \cdot ; \cdot)$ denotes the hypergeometric function [35].

Proof: Please refer to Appendix III.

Because of the complexity of the statistical characteristics of the product of multiple SR fading variables, there exists an integral operation in (25). However, it can be easily solved by the built-in function in numerical software, such as int in MATLAB. So, the obtained analytical result can readily serve as a useful tool to study the outage performance of the HARQ-IR scheme in STT scenarios.

Therefore, the OP for the whole system under the GSA scheme can be obtained by substituting (25) into (12).

\section{Diversity GAIN}

Clearly, HARQ schemes exploit either coding gain or time diversity to improve the reliability of the transmissions between the communication pairs. In this work, the diversity order of the considered system 
is given by

$$
\Delta=-\lim _{\bar{\lambda} \rightarrow \infty} \frac{\log P_{\text {out }, i j}}{\log \bar{\lambda}}
$$

where $\bar{\lambda}$ is defined after (7).

\section{A. GSA Scheme}

Lemma 1. The diversity order of the considered system under GSA scheme is unitary.

Proof: The OP under GSA scheme can be written as

$$
\operatorname{Pr}\left\{\lambda_{j} \leq \varepsilon d_{i}^{n}\right\}=\mathbb{E}_{d_{i}}\left\{F_{\lambda_{j}}\left(\varepsilon d_{i}^{n}\right)\right\}
$$

From [36, Eq. (8)], the asymptotic CDF of $\lambda_{j}$ is given by

$$
F_{\lambda_{j}}(x) \stackrel{\bar{\lambda}}{\rightarrow} \underset{\simeq}{\simeq} \alpha x \bar{\lambda}^{-1}
$$

Substituting (28) into (27) yields

$$
P_{\text {out }, i j}=\operatorname{Pr}\left\{\lambda_{j} \leq \varepsilon d_{i}^{n}\right\} \stackrel{\bar{\lambda} \rightarrow \infty}{\simeq} \bar{\lambda}^{-1} \mathbb{E}_{d_{i}}\left\{\alpha\left(\varepsilon d_{i}^{n}\right)\right\}
$$

From the definition of diversity order in (26), the corresponding diversity order of the OP under GSA scheme is easily obtained as

$$
\Delta_{\mathrm{GSA}}=-\lim _{\bar{\lambda} \rightarrow \infty} \frac{\log \left(\bar{\lambda}^{-1}\right)+\log \mathbb{E}_{d_{i}}\left\{\alpha\left(\varepsilon d_{i}^{n}\right)\right\}}{\log \bar{\lambda}}=1
$$

which completes the proof.

\section{B. RTD Scheme}

Lemma 2. The diversity order of the considered system under RTD scheme is $\mathcal{M}$.

Proof: We first investigate the diversity order of the CDF of $\max \left\{\lambda_{1}, \lambda_{2}, \cdots, \lambda_{\mathcal{M}}\right\} d_{i}^{-n}$. It is easy to obtain the $\mathrm{CDF}$ of $\max \left\{\lambda_{1}, \lambda_{2}, \cdots, \lambda_{\mathcal{M}}\right\} d_{i}^{-n}$, given by

$$
\operatorname{Pr}\left\{\max \left\{\lambda_{1}, \lambda_{2}, \cdots, \lambda_{\mathcal{M}}\right\} \leq x d_{i}^{n}\right\}=\mathbb{E}_{d_{i}}\left\{\left[F_{\lambda_{1}}(x)\right]^{\mathcal{M}}\right\}^{\stackrel{\bar{\lambda}}{\simeq}{ }^{\infty}} \bar{\lambda}^{-\mathcal{M}} \mathbb{E}_{d_{i}}\left\{\left(\alpha x d_{i}^{n}\right)^{\mathcal{M}}\right\},
$$

The corresponding diversity order is

$$
\Delta=-\lim _{\bar{\lambda} \rightarrow \infty} \frac{\log \left(\bar{\lambda}^{-\mathcal{M}}\right)+\log \mathbb{E}_{d_{i}}\left\{\left(\alpha x d_{i}^{n}\right)^{\mathcal{M}}\right\}}{\log \bar{\lambda}}=\mathcal{M} .
$$


Then, we can easily see that $\mathcal{M} \max \left\{\lambda_{1}, \lambda_{2}, \cdots, \lambda_{\mathcal{M}}\right\} d_{i}^{-n}$ has the same order as $\max \left\{\lambda_{1}, \lambda_{2}, \cdots, \lambda_{\mathcal{M}}\right\} d_{i}^{-n}$. Finally, in view of the following relationship,

$$
\begin{aligned}
\frac{\max \left\{\lambda_{1}, \lambda_{2}, \cdots, \lambda_{\mathcal{M}}\right\}}{d_{i}^{n}} & \leq \frac{\lambda_{1}+\lambda_{2}+\cdots+\lambda_{\mathcal{M}}}{d_{i}^{n}} \\
& \leq \frac{\mathcal{M} \max \left\{\lambda_{1}, \lambda_{2}, \cdots, \lambda_{\mathcal{M}}\right\}}{d_{i}^{n}}
\end{aligned}
$$

we come to the conclusion that the diversity order of the OP under RTD scheme is $\mathcal{M}$.

\section{HRAQ-IR Scheme}

Lemma 3. The diversity order of the considered system under HRAQ-IR scheme is $\mathcal{M}$.

Proof: Let $X_{i}=\bar{\lambda}\left|h_{\mathrm{SD} i}[s]\right|^{2}$. It is obvious that the OP under HRAQ-IR scheme is the CDF of $\left(X_{1} X_{2} \cdots X_{\mathcal{M}}\right) d_{i}^{-n \mathcal{M}}$, which means that we can alternatively calculate the diversity order of the CDF of $\left(X_{1} X_{2} \cdots X_{\mathcal{M}}\right) d_{i}^{-n \mathcal{M}}$.

First, the CDF of $\frac{\left[\min \left\{X_{1} X_{2} \cdots X_{\mathcal{M}}\right\}\right]^{\mathcal{M}}}{d_{i}^{n \mathcal{M}}}$ can be derived as

$$
\begin{aligned}
\operatorname{Pr}\left\{\frac{\left[\min \left\{X_{1} X_{2} \cdots X_{\mathcal{M}}\right\}\right]^{\mathcal{M}}}{d_{i}^{n \mathcal{M}}} \leq x\right\} & =1-\operatorname{Pr}\left\{\min \left\{X_{1} X_{2} \cdots X_{\mathcal{M}}\right\}>d_{i}^{n} x^{\frac{1}{\mathcal{M}}}\right\} \\
& =1-\mathbb{E}_{d_{i}}\left\{\left[1-F_{X_{1}}\left(d_{i}^{n} x^{\frac{1}{\mathcal{M}}}\right)\right]^{\mathcal{M}}\right\} .
\end{aligned}
$$

By using $1-(1-x)^{\mathcal{M}} \stackrel{x \rightarrow 0}{\approx} x^{\mathcal{M}}$ and the asymptotic CDF of $X_{1}\left(F_{X_{1}}(x) \approx \alpha \bar{\lambda}^{-1} x\right.$ for $\bar{\lambda} \rightarrow \infty$ [36]), the probability can be further written as

$$
\begin{aligned}
\operatorname{Pr}\left\{\frac{\left[\min \left\{X_{1} X_{2} \cdots X_{\mathcal{M}}\right\}\right]^{\mathcal{M}}}{d_{i}^{n \mathcal{M}}} \leq x\right\} & \approx \mathbb{E}_{d_{i}}\left\{\left[F_{X_{1}}\left(d_{i}^{n} x^{\frac{1}{\mathcal{M}}}\right)\right]^{\mathcal{M}}\right\}=\mathbb{E}_{d_{i}}\left\{\left[\alpha \bar{\lambda}^{-1} d_{i}^{n} x^{\frac{1}{\mathcal{M}}}\right]^{\mathcal{M}}\right\} \\
& =\bar{\lambda}^{-\mathcal{M}} \mathbb{E}_{d_{i}}\left\{\left[\alpha d_{i}^{n} x^{\frac{1}{\mathcal{M}}}\right]^{\mathcal{M}}\right\} .
\end{aligned}
$$

The corresponding diversity order can be easily derived as

$$
\begin{aligned}
\Delta_{\text {min }} & =-\lim _{\bar{\lambda} \rightarrow \infty} \frac{\log \operatorname{Pr}\left\{\frac{\left[\min \left\{X_{1} X_{2} \cdots X_{\mathcal{M}}\right\}\right]^{\mathcal{M}}}{d_{i}^{n \mathcal{M}}} \leq x\right\}}{\log \bar{\lambda}} \\
& =-\lim _{\bar{\lambda} \rightarrow \infty} \frac{\log \left(\bar{\lambda}^{-\mathcal{M}}\right)+\log \mathbb{E}_{d_{i}}\left\{\left[\alpha d_{i}^{n} x^{\frac{1}{\mathcal{M}}}\right]^{\mathcal{M}}\right\}}{\log \bar{\lambda}} \\
& =\mathcal{M} .
\end{aligned}
$$


Then, the CDF of $\frac{\left[\max \left\{X_{1} X_{2} \cdots X_{\mathcal{M}}\right\}\right]^{\mathcal{M}}}{d_{i}^{n \mathcal{M}}}$ is given by

$$
\operatorname{Pr}\left\{\frac{\left[\max \left\{X_{1} X_{2} \cdots X_{\mathcal{M}}\right\}\right]^{\mathcal{M}}}{d_{i}^{n \mathcal{M}}} \leq x\right\}=\mathbb{E}_{d_{i}}\left\{\left[F_{X_{1}}\left(d_{i}^{n} x^{\frac{1}{\mathcal{M}}}\right)\right]^{\mathcal{M}}\right\}^{\bar{\lambda} \rightarrow^{\infty}} \bar{\lambda}^{-\mathcal{M}} \mathbb{E}_{d_{i}}\left\{\left[\alpha d_{i}^{n} x^{\frac{1}{\mathcal{M}}}\right]^{\mathcal{M}}\right\}
$$

which implies that the corresponding diversity order is $\Delta_{\max }=\mathcal{M}$.

Finally, according to the following relationship,

$$
\begin{aligned}
\frac{\left[\min \left\{X_{1} X_{2} \cdots X_{\mathcal{M}}\right\}\right]^{\mathcal{M}}}{d_{i}^{n \mathcal{M}}} & \leq \frac{X_{1} X_{2} \cdots X_{\mathcal{M}}}{d_{i}^{n \mathcal{M}}} \\
& \leq \frac{\left[\max \left\{X_{1} X_{2} \cdots X_{\mathcal{M}}\right\}\right]^{\mathcal{M}}}{d_{i}^{n \mathcal{M}}}
\end{aligned}
$$

one can conclude that the diversity order of the OP under HRAQ-IR scheme is $\mathcal{M}$.

\section{NumericAl Results}

In this section, numerical results will be provided to study the outage performance of the considered HARQ schemes, as well as to verify the proposed analytical models. In the following, we use D instead of $\mathrm{D}_{i}$ for simplification, and the path-loss factor for the S-D link is set as 2 . Furthermore, we run $1 \times 10^{5}$ times of the realizations of the considered system and $1 \times 10^{6}$ trials of Monte-Carlo simulations, to model the randomness of the positions of the considered terminals and channel gains over each link. Unless otherwise explicitly specified, the main parameters adopted in this section are set as $n=2, R=0.01$ bps, $K=10, \mathcal{M}=10, H=300 \mathrm{~km}, L=50 \mathrm{~km}, m=3, b=2.56, \Omega=0.123$.

\section{A. Outage Performance under GSA and RTD Schemes}

In this subsection, the outage performance of GSA and RTD schemes in STT scenarios will be studied, while $L=20 \mathrm{~km}$.

In Figs. 2 and 3, the OP under GSA scheme is presented for various $m$ and $H$, respectively, while the one under RTD scheme is given in Figs. 4 and 5.

It is easy to see from Figs. 2 and 4 that the OP under GSA and RTD schemes with a large $m$ outperforms the one with a low $m$. This observation can be explained by the fact that a large $m$ represents fewer obstructions of the LOS components between the satellite and the terrestrial receivers. In other words, a large $m$ means a better channel condition for the transmissions over STT links.

In Figs. 3 and 5, the OP under GSA and RTD schemes is respectively depicted for various values of $H$ from $300 \mathrm{~km}$ to $400 \mathrm{~km}$. Clearly, the OP under the GSA scheme degrades with the increment of $H$. That 


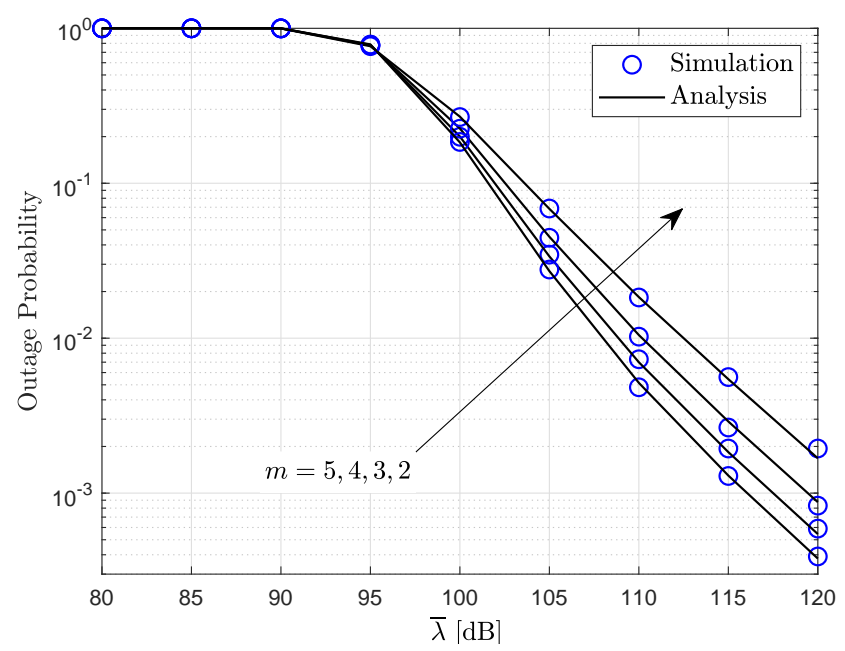

Fig. 2: Outage probability versus transmit SNR $\bar{\lambda}$ for various values of the fading severity parameter $m$ under GSA scheme.

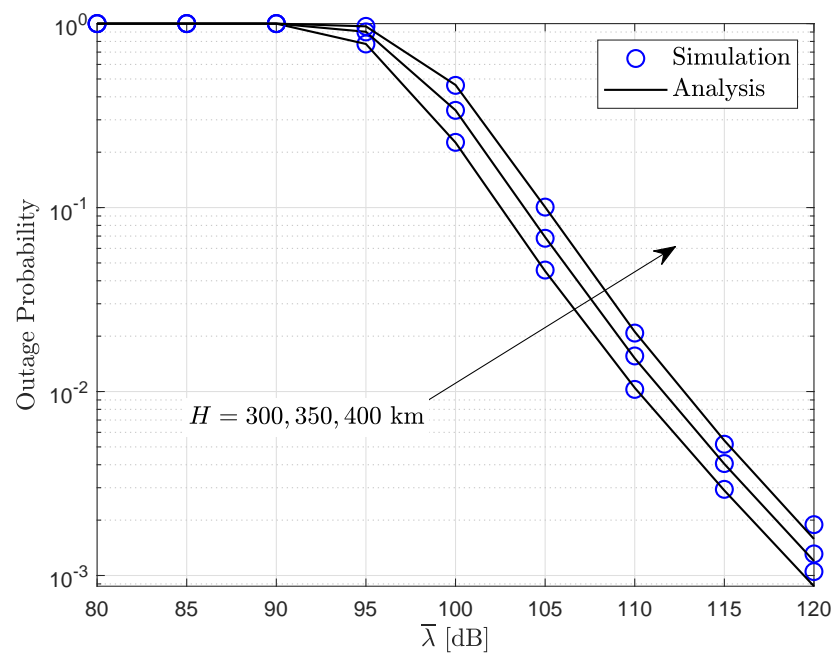

Fig. 3: Outage probability versus transmit SNR $\bar{\lambda}$ for various values of the satellite altitude $H$ under GSA scheme.

is to say, the OP of the GSA scheme gets worse while the height of the orbit enlarges, which leads to increased path-loss. Recalling the truth that, compared with small-scale fading, path-loss mainly domains the fading effects suffered by the transmitted signals when the transmission distance is large enough, the finding here is easy to understand from the fact that in STT scenarios the transmission distance ranges from hundreds to thousands of kilometers. 


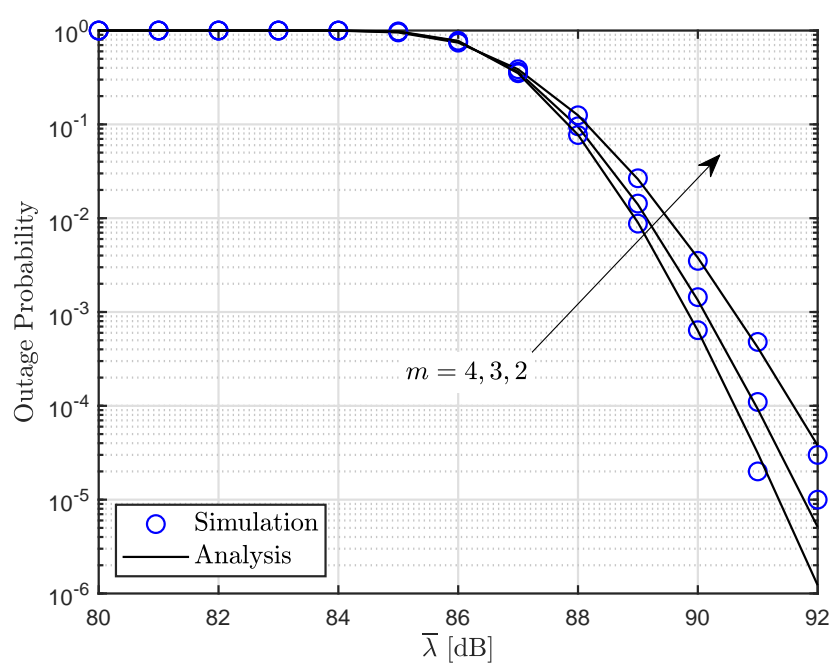

Fig. 4: Outage probability versus transmit $\operatorname{SNR} \bar{\lambda}$ for various values of the fading severity parameter $m$ under RTD scheme.

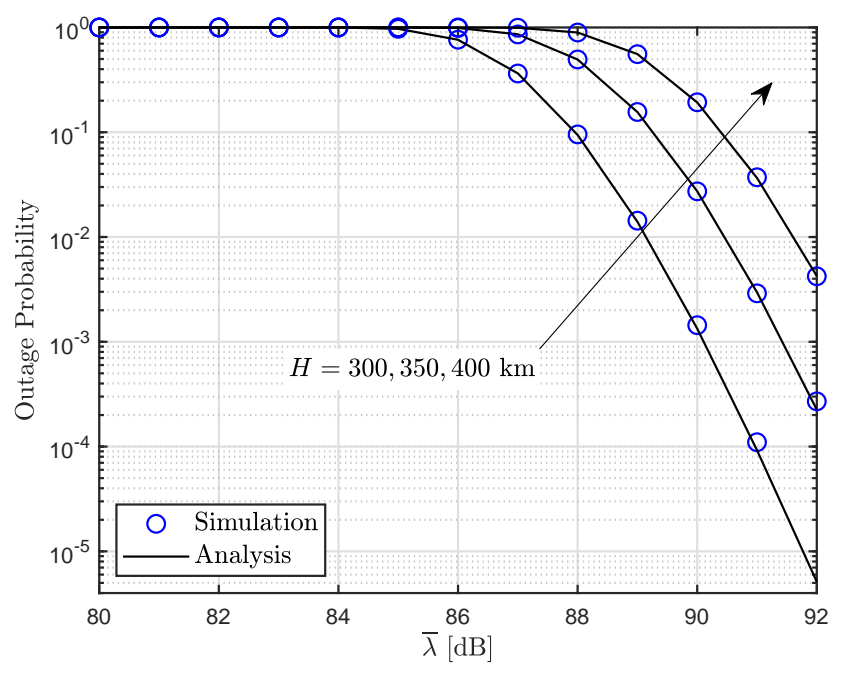

Fig. 5: Outage probability versus transmit SNR $\bar{\lambda}$ for various values of the satellite altitude $H$ under RTD scheme.

Furthermore, one can also see that the outage performance under both schemes can be improved, while the transmit SNR, $\bar{\lambda}$, increases, which indicates that the received SNR at the terrestrial receiver can be improved. 


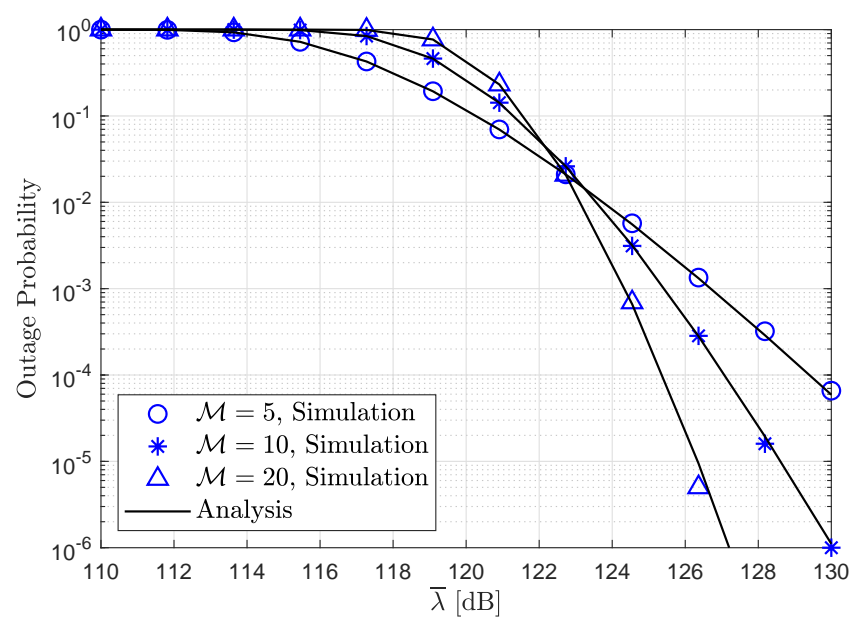

Fig. 6: Outage probability versus transmit $\operatorname{SNR} \bar{\lambda}$ for various values of $\mathcal{M}$ under HARQ-IR scheme for $2^{R}=0.01, m=2.56, b=0.0158, \Omega=8.97 \times 10^{-4}$ (i.e., infrequent light shadowing).

The outage performance of the HARQ-IR scheme will be investigated in this subsection while considering the effects of the randomness of small-scale fading and the positions of the terrestrial receivers.

Observing the principles achieved in the previous subsection, we can readily reach some similar conclusions with the HARQ-IR scheme, namely, the ones addressing the effects of $m, H$, and $\bar{\lambda}$ on the outage performance, as the same transmission scenario shown in Fig. 1 is considered for these three schemes. Therefore, to more completely understand how the system parameters affect the outage performance of the target system and save page space, the effects of some other system parameters, i.e., $\mathcal{M}$ and $R$, will be studied in this subsection, instead of considering the cases of different $m$ and $H$.

Obviously, the OP under HARQ-IR scheme with a low $\mathcal{M}$ outperforms the one with a large $\mathcal{M}$ when $\bar{\lambda}$ is less than $123 \mathrm{~dB}$. On the contrary, an opposite observation is found while $\bar{\lambda}$ is larger than 123 $\mathrm{dB}$. What is presented here means that, when $\bar{\lambda}$ is not large enough, the outage performance under the HARQ-IR scheme cannot be improved via exploiting more copies of the transmitted signal during the decoding process.

As presented in Fig. 7, the influence of the outage threshold, $R$, is investigated. As expected, enlarging $R$ shows a negative effect on the OP of the HARQ-IR scheme, since a large outage threshold represents a large probability of outage events. Furthermore, we can also conclude that the outage threshold exhibits a similar effect on the outage performance of the two other schemes, GSA and RTD schemes. 


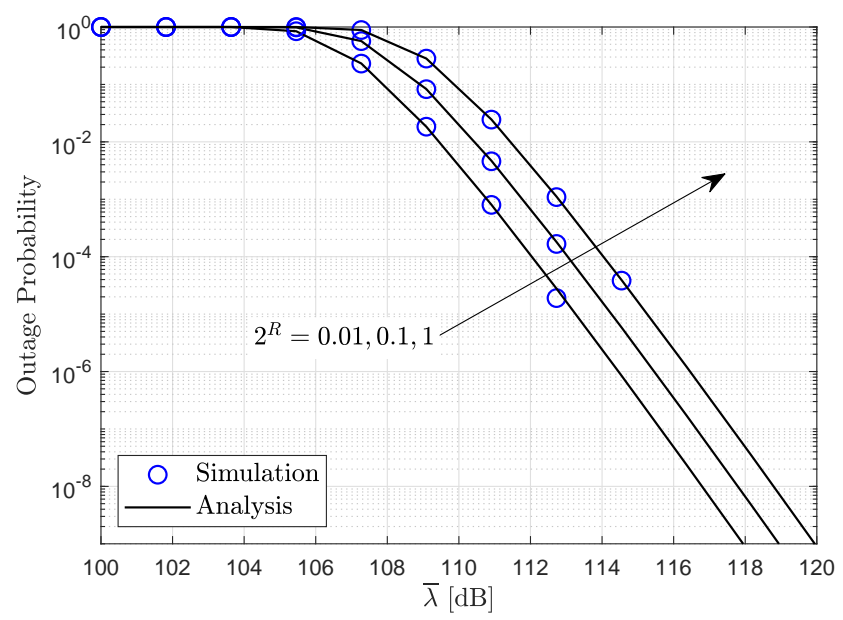

Fig. 7: Outage probability versus transmit SNR $\bar{\lambda}$ for various values of $R$ under HARQ-IR scheme for $m=19.4, b=0.158, \Omega=1.29$ (i.e., infrequent light shadowing).

Further, one can easily see from Figs. 2-7 that simulation and numerical results can match with each other well in various cases, which verify the correctness of our proposed analytical models.

\section{Comparisons Among The Three Schemes}

In this subsection, we will compare the outage performance of these three schemes in various cases.

As depicted in Figs. 8 and 9, RTD, and HARQ-IR schemes with a same $\mathcal{M}$ show the same outage performance, while outperforming GSA scheme. Because the diversity gain in the time domain, which is shown in (19) and (23), can be achieved under both RTD and HARQ-IR schemes, leading to improved outage performance, while only one copy of the received signal is adopted for decoding under GSA scheme, as suggested by (16).

Moreover, recalling (7), (19), and (23), one can have $\log _{2}\left(1+\sum_{s=(j-M+1)^{+}}^{j} \gamma_{i s}\right) \approx \sum_{s=(j-M+1)^{+}}^{j} \log _{2}\left(1+\gamma_{i s}\right)$ while $\bar{\lambda}$ is sufficiently large, which can be used to explain the finding that RTD and HARQ-IR schemes with a same $\mathcal{M}$ exhibit similar outage performance in Figs. 8 and 9. This finding can also be verified by the theoretical analysis on the diversity gain presented in Section IV, namely, the diversity gains of RTD and HARQ-IR schemes are $\mathcal{M}$, as suggested by Lemma 2 and 3.

As the three different kinds of HARQ schemes exhibit different outage performance and require different levels of resource consumption, they can be chosen and applied in practical applications by considering 


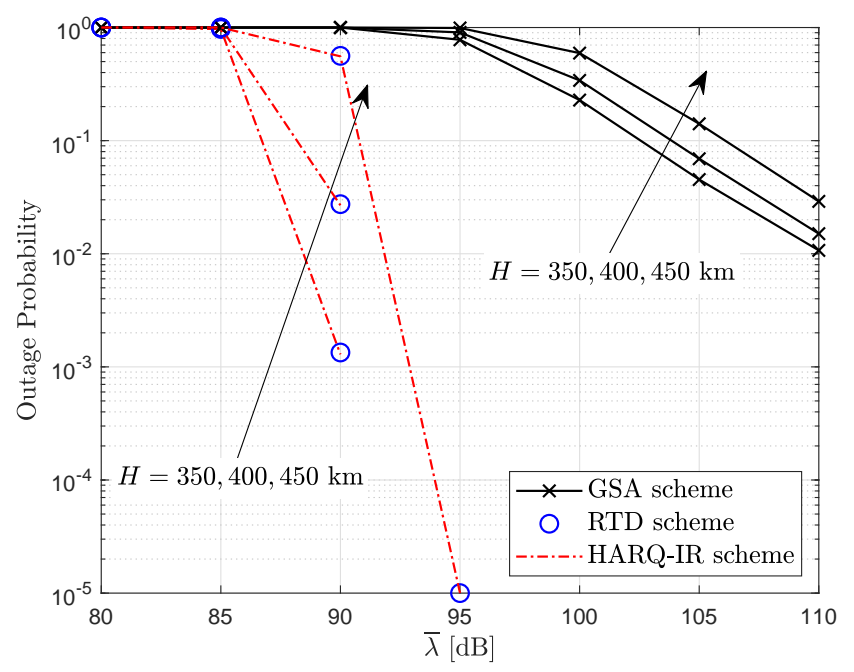

Fig. 8: Outage probability for various values of the satellite altitude $H$ under the three schemes.

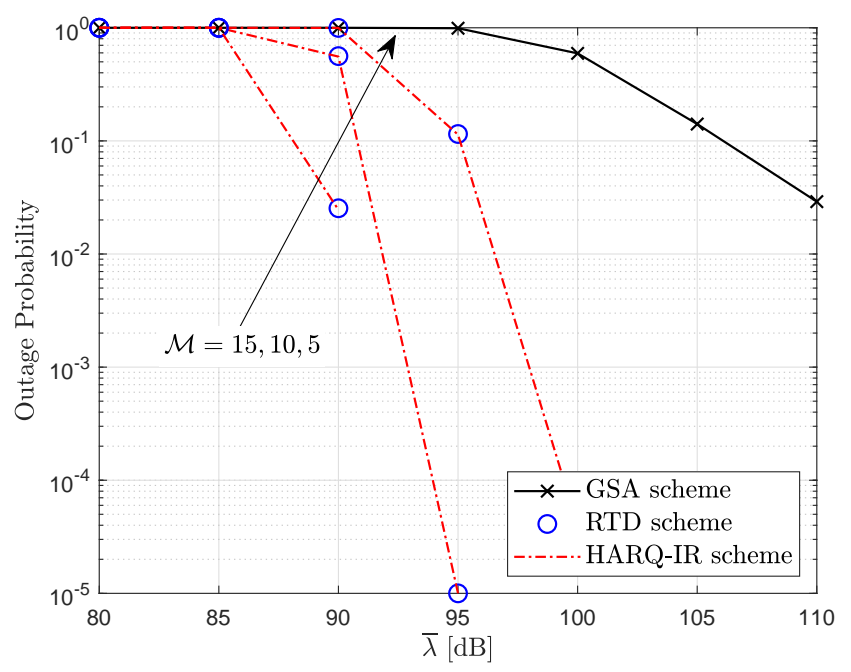

Fig. 9: Outage probability for various values of $\mathcal{M}$ under the three schemes with $H=450 \mathrm{~km}$.

the hardware resources at terrestrial receivers, e.g., power budgets and computational capability, and the application requirements, e.g., the working lifetime of the terrestrial receivers.

\section{CONCLUSION}

In this paper, we mainly study the outage performance and diversity gain under three different HARQ schemes, GSA, RTD, and HARQ-IR schemes, in STT scenarios, while considering the randomness of 
the power gain of the wireless channels and the positions of the terrestrial receivers. Analytical modes are presented for the OP under these three schemes, via which some useful conclusions are obtained as follows:

1. Path-loss plays a key role in affecting the outage performance of these HARQ schemes, compared with small-scale fading over STT channels;

2. The transmission distance shows a negative effect on the outage performance, while the shape parameter, $m$, of SR channel, exhibits a positive influence on the outage performance;

3. When the transmit SNR is sufficiently large, RTD and HARQ-IR schemes are with similar outage performance;

4. The outage performance of both RTD and HARQ-IR schemes outperforms the one of GSA, profiting from the adopted diversity gain in the time domain;

5. The diversity orders of GSA, RTD, and HARQ-IR schemes are $1, \mathcal{M}$, and $\mathcal{M}$, respectively.

In this work, we only focus on the impacts of channel fading and the distribution of terrestrial receivers on the outage performance of HARQ schemes. In the future, the continuity of the transmission behaviors on the timeline will be considered to further understand the performance of these HARQ schemes.

\section{APPENDIX I: PROOF OF THEOREM 2}

Considering the randomness of $\lambda_{j}$ and using (9), the OP under GSA scheme in the $j$ th $(1 \leq j \leq K)$ time slot can be rewritten as

$$
\begin{aligned}
P_{\text {out }, i j} & =\operatorname{Pr}\left\{\lambda_{j}<\varepsilon d_{i}^{n}\right\} \\
& =1-\alpha \sum_{k=0}^{m-1} \frac{\varsigma(k)}{\bar{\lambda}^{k+1}} \sum_{p=0}^{k} \frac{k !}{p !}\left(\frac{\beta-\delta}{\bar{\lambda}}\right)^{-(k+1-p)} \varepsilon^{p} d_{i}^{n p} \exp \left(-\frac{\varepsilon(\beta-\delta)}{\bar{\lambda}} d_{i}^{n}\right) .
\end{aligned}
$$

Then, taking the randomness of the position of $\mathrm{D}_{i}$ into account, the OP under GSA scheme in the $j$ th $(1 \leq j \leq K)$ time slot can be finally calculated as

$$
\begin{aligned}
P_{\text {out }, i j} & =1-\frac{2 \alpha}{L^{2}} \sum_{k=0}^{m-1} \frac{\varsigma(k)}{\bar{\lambda}^{k+1}} \sum_{p=0}^{k} \frac{k !}{p !}\left(\frac{\beta-\delta}{\bar{\lambda}}\right)^{-(k+1-p)} \varepsilon^{p} \int_{H_{1}}^{\sqrt{H_{1}{ }^{2}+L^{2}}} x^{n p+1} \exp \left(-\frac{\varepsilon(\beta-\delta)}{\bar{\lambda}} x^{n}\right) d x \\
& \stackrel{(a)}{=} 1-\frac{2 \alpha}{n L^{2}} \sum_{k=0}^{m-1} \frac{\varsigma(k)}{\bar{\lambda}^{k+1}} \sum_{p=0}^{k} \frac{k !}{p !}\left(\frac{\beta-\delta}{\bar{\lambda}}\right)^{-\left(k+\frac{2}{n}+1\right)} \varepsilon^{-\frac{2}{n}} \int_{y_{\min }}^{y_{\max }} y^{p+\frac{2}{n}-1} \exp (-y) d y \\
& =1-\frac{2 \alpha}{n L^{2}} \sum_{k=0}^{m-1} \frac{\varsigma(k)}{\bar{\lambda}^{k+1}} \sum_{p=0}^{k} \frac{k !}{p !}\left(\frac{\beta-\delta}{\bar{\lambda}}\right)^{-\left(k+\frac{2}{n}+1\right)} \varepsilon^{-\frac{2}{n}}\left[\gamma\left(p+\frac{2}{n}, y_{\max }\right)-\gamma\left(p+\frac{2}{n}, y_{\min }\right)\right]
\end{aligned}
$$


where $(a)$ denotes $y=\frac{\varepsilon(\beta-\delta)}{\lambda} x^{n} \Rightarrow x=y^{\frac{1}{n}}\left(\frac{\varepsilon(\beta-\delta)}{\lambda}\right)^{-\frac{1}{n}} \Rightarrow d x=\frac{1}{n}\left(\frac{\varepsilon(\beta-\delta)}{\lambda}\right)^{-\frac{1}{n}} y^{\frac{1}{n}-1} d y, y_{\min }=$ $\frac{\varepsilon(\beta-\delta)}{\bar{\lambda}} H_{1}^{n}, y_{\max }=\frac{\varepsilon(\beta-\delta)}{\bar{\lambda}}\left(H_{1}{ }^{2}+L^{2}\right)^{\frac{n}{2}}$, and $\gamma(a, x)=\int_{0}^{x} t^{a-1} \exp (-t) d t$ is the lower incomplete gamma function.

With this result, the proof of Theorem 2 is completed.

\section{APPENDIX II: PROOF OF THEOREM 3}

As suggested in [37], the CDF of $\Lambda=\sum_{s=(j-M+1)^{+}}^{j} \lambda_{s}$ can be given as

$$
F_{\Lambda}(x)=\alpha^{\mathcal{M}} \sum_{l=0}^{c}\left(\begin{array}{l}
c \\
l
\end{array}\right) \beta^{c-l}[G(x, l, d)+\nu \delta G(x, l, d+1)],
$$

where $\mathcal{M}=j-[j-M]^{+}, c=[d-\mathcal{M}]^{+}, \nu=m \mathcal{M}-d, d=\max \{\mathcal{M},\lfloor m \mathcal{M}\rfloor\},\lfloor x\rfloor$ denotes the largest integer not larger than $x,[x]^{+}$indicates that if $x \leq 0$, then use $x=0$, and

$$
G(x, l, d)=\frac{(\beta-\delta)^{\frac{l-d-1}{2}}}{\bar{\lambda}^{\frac{d-l-1}{2}} \Gamma(d-l-1)} x^{\frac{d-l-1}{2}} \exp \left(-\frac{\beta-\delta}{2 \bar{\lambda}} x\right) M_{\frac{d-l-1}{2}, \frac{d-l}{2}}\left(\frac{\beta-\delta}{\bar{\lambda}} x\right),
$$

where $M_{\lambda, \mu}(z)$ is Whittaker functions, as defined as [35, Eq. (9.220.2)].

Using [38, Eq. (8.4.43.1)], $G(x, l, d)$ can be rewritten as

$$
G(x, l, d)=\frac{(\beta-\delta)^{\frac{l-d-1}{2}}}{\bar{\lambda}^{\frac{d-l-1}{2}} \Gamma(d)} x^{\frac{d-l-1}{2}} G_{1,2}^{1,1}\left[\begin{array}{l|c}
\frac{\beta-\delta}{\bar{\lambda}} x & \frac{3-d-l}{2} \\
\frac{d-l+1}{2}, \frac{1-d+l}{2}
\end{array}\right],
$$

where $G_{p, q}^{m, n}\left[x \mid \begin{array}{l}a_{1}, \cdots, a_{p} \\ b_{1}, \cdots, b_{q}\end{array}\right]$ is the Meijer's $G$-function, as defined by [35, Eq. (9.301)].

Considering the randomness of $\Lambda=\sum_{s=(j-M+1)^{+}}^{j} \lambda_{s}$, the OP under RTD scheme in the $j$ th $(1 \leq j \leq K)$ time slot can be further written as

$$
\begin{aligned}
P_{\text {out }, i j} & =\operatorname{Pr}\left\{\sum_{s=(j-M+1)^{+}}^{j} \lambda_{s}<\varepsilon d_{i}^{n}\right\} \\
& =\alpha^{\mathcal{M}} \sum_{l=0}^{c}\left(\begin{array}{l}
c \\
l
\end{array}\right) \beta^{c-l}\left[G\left(\varepsilon d_{i}^{n}, l, d\right)+\nu \delta G\left(\varepsilon d_{i}^{n}, l, d+1\right)\right] .
\end{aligned}
$$

Next, taking the randomness of the position of $\mathrm{D}_{i}$ into account, the OP under RTD scheme in the $j$ th 
$(1 \leq j \leq K)$ time slot can be calculated as

$$
\begin{aligned}
& P_{\text {out }, i j}=\int_{H_{1}}^{\sqrt{H_{1}{ }^{2}+L^{2}}} \alpha^{\mathcal{M}} \sum_{l=0}^{c}\left(\begin{array}{l}
c \\
l
\end{array}\right) \beta^{c-l}\left[G\left(\varepsilon d_{i}^{n}, l, d\right)+\nu \delta G\left(\varepsilon d_{i}^{n}, l, d+1\right)\right] f_{d_{i}}(x) d x \\
& =\alpha^{\mathcal{M}} \sum_{l=0}^{c}\left(\begin{array}{l}
c \\
l
\end{array}\right) \beta^{c-l}[\underbrace{\int_{H_{1}}^{\sqrt{H_{1}{ }^{2}+L^{2}}} G\left(\varepsilon d_{i}^{n}, l, d\right) f_{d_{i}}(x) d x}_{I_{1}}+\nu \delta \underbrace{\int_{H_{1}}^{\sqrt{H_{1}^{2}+L^{2}}} G\left(\varepsilon d_{i}^{n}, l, d+1\right) f_{d_{i}}(x) d x}_{I_{2}}]
\end{aligned}
$$

Using (4) and [39, Eq. (26)], $I_{1}$ can be derived as

$$
\begin{aligned}
& I_{1}=\int_{H_{1}}^{\sqrt{H_{1}^{2}+L^{2}}} G\left(\varepsilon d_{i}^{n}, l, d\right) f_{d_{i}}(x) d x \\
& =\frac{2(\beta-\delta)^{\frac{l-d-1}{2}}}{L^{2} \Gamma(d)}\left(\frac{\varepsilon}{\bar{\lambda}}\right)^{\frac{d-l-1}{2}} \int_{H_{1}}^{\sqrt{H_{1}^{2}+L^{2}}} x^{\frac{n(d-l-1)}{2}+1} G_{1,2}^{1,1}\left[\frac{\varepsilon(\beta-\delta)}{\bar{\lambda}} x^{n} \mid \begin{array}{c}
\frac{3-d-l}{2} \\
\frac{d-l+1}{2}, \frac{1-d+l}{2}
\end{array}\right] d x \\
& \stackrel{(b)}{=} \frac{2(\beta-\delta)^{\frac{l-d-1}{2}}}{n L^{2} \Gamma(d)}\left(\frac{\varepsilon}{\bar{\lambda}}\right)^{\frac{d-l-1}{2}} \int_{t_{\min }}^{t_{\max }} t^{\frac{d-l-1}{2}+\frac{2}{n}-1} G_{1,2}^{1,1}\left[\begin{array}{c|c}
\frac{\varepsilon(\beta-\delta)}{\bar{\lambda}} t & \frac{3-d-l}{2} \\
\frac{d-l+1}{2}, \frac{1-d+l}{2}
\end{array}\right] d t
\end{aligned}
$$

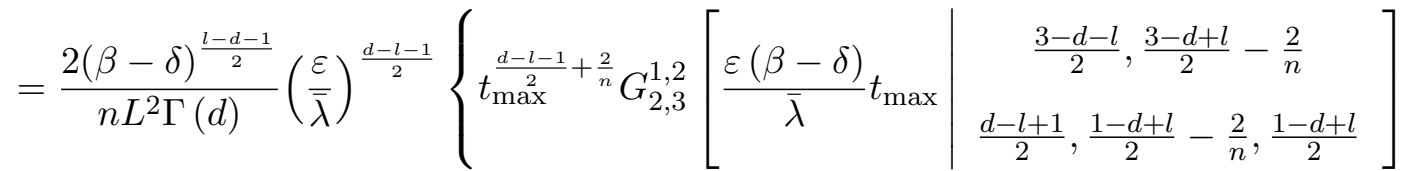

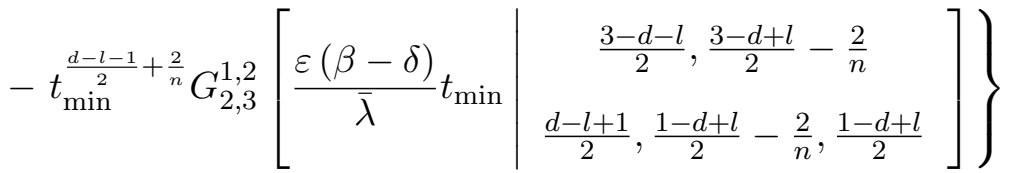

where $(b)$ denotes $t=x^{n} \Rightarrow x=t^{\frac{1}{n}} \Rightarrow d x=\frac{1}{n} t^{\frac{1}{n}-1} d t, t_{\max }=\left(H_{1}{ }^{2}+L^{2}\right)^{\frac{n}{2}}$, and $t_{\min }=H_{1}{ }^{n}$.

Similarly, $I_{2}$ in (45) can also be calculated as

$$
\begin{aligned}
& I_{2}=\int_{H_{1}}^{\sqrt{H_{1}^{2}+L^{2}}} G\left(\varepsilon d_{i}^{n}, l, d+1\right) f_{d_{i}}(x) d x
\end{aligned}
$$

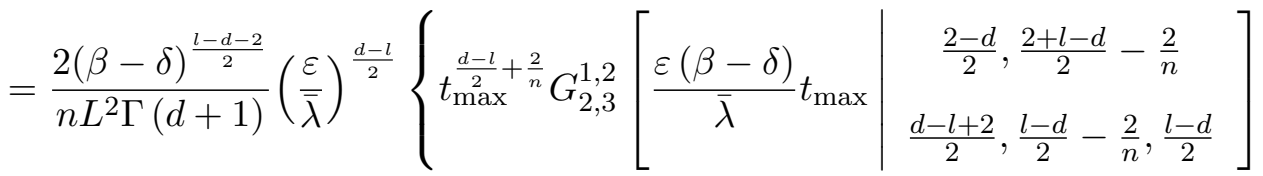

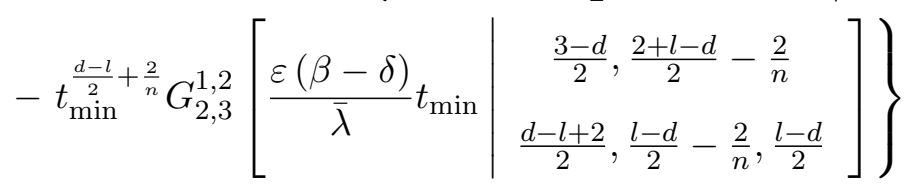


Finally, the OP under RTD scheme in the $j$ th $(1 \leq j \leq K)$ time slot can be achieved as (21) by substituting (46) and (47) into (45).

\section{APPENDIX III: PROOF OF THEOREM 4}

First, we calculate the complex moment of $\left|h_{\mathrm{SD}_{i}}[s]\right|^{2}$. By using the PDF of $\left|h_{\mathrm{SD}_{i}}[s]\right|^{2}$, we can have $\Theta=\mathbb{E}\left\{\left(\left|h_{\mathrm{SD}_{i}}[s]\right|^{2}\right)^{j t}\right\}$ as

$$
\begin{aligned}
\Theta & =\alpha \int_{0}^{\infty} x^{\jmath t} \exp (-\beta x) \cdot{ }_{1} F_{1}(m ; 1 ; \delta x) d x \\
& =\alpha \beta^{-(\jmath t+1)} \Gamma(\jmath t+1) \cdot{ }_{2} F_{1}\left(m, \jmath t+1 ; 1 ; \frac{\delta}{\beta}\right), \quad t \geq 0 .
\end{aligned}
$$

Then, by using (4), the PDF of $Z=\frac{d_{i}^{n \mathcal{M}}}{\lambda^{\mathcal{M}}}\left(z_{\min } \leq z \leq z_{\max }\right)$ can be derived as

$$
f_{Z}(z)=\frac{\bar{\lambda}^{\mathcal{M}}}{n \mathcal{M}} \frac{2}{L^{2}}\left(\bar{\lambda}^{\mathcal{M}} z\right)^{\frac{2}{n \mathcal{M}}-1}=\frac{2 \bar{\lambda}^{\frac{2}{n}}}{n L^{2} \mathcal{M}} z^{\frac{2}{n \mathcal{M}}-1} .
$$

The complex $(-\jmath t)$-th moment of $Z$ is

$$
\begin{aligned}
\mathbb{E}\left\{Z^{-\jmath t}\right\} & =\frac{2 \bar{\lambda}^{\frac{2}{n}}}{n \mathcal{M} L^{2}} \int_{z_{\min }}^{z_{\max }} z^{\frac{2}{n \mathcal{M}}-\jmath t-1} d z \\
& =\jmath \frac{2 \bar{\lambda}^{\frac{2}{n}}}{L^{2}} \frac{z_{\max }^{\frac{2}{n \mathcal{M}}-\jmath t}-z_{\min }^{\frac{2}{n \mathcal{M}}-\jmath t}}{2 \jmath+n \mathcal{M} t} .
\end{aligned}
$$

Further, by considering the virtues of logarithm function, let $W^{\prime}=\log \left(\frac{Y}{Z}\right)$. The characteristic function (CF) of $W^{\prime}$ can be derived as

$$
\begin{aligned}
\mathrm{CF}_{W^{\prime}}(t) & =\mathbb{E}\left\{\exp \left(\jmath t \log \left(\frac{\prod_{s=(j-\mathcal{M}+1)^{+}}^{j}\left|h_{\mathrm{SD}_{i}}[s]\right|^{2}}{Z}\right)\right)\right\} \\
& =\left[\mathbb{E}\left\{\left(\left|h_{\mathrm{SD}_{i}}[s]\right|^{2}\right)^{\jmath t}\right\}\right]^{\mathcal{M}} \mathbb{E}\left\{Z^{-\jmath t}\right\} .
\end{aligned}
$$

According to Gil-Pelaez Theorem, the CDF of $W^{\prime}$ can be deduced from the CF of $W^{\prime}$, given by

$$
F_{W^{\prime}}\left(w^{\prime}\right)=\frac{1}{2}-\frac{1}{\pi} \int_{0}^{\infty} \frac{\operatorname{Im}\left\{\exp \left(-\jmath t w^{\prime}\right) \mathrm{CF}_{W^{\prime}}(t)\right\}}{t} d t .
$$

It is easy to see that the relationship between the CDFs of $W$ and $W^{\prime}$ is

$$
\begin{aligned}
F_{W}(w) & =\operatorname{Pr}\{\log W \leq \log w\}=F_{W^{\prime}}(\log w) \\
& =\frac{1}{2}-\frac{1}{\pi} \int_{0}^{\infty} \frac{\operatorname{Im}\left\{w^{-\jmath t} \mathrm{CF}_{W^{\prime}}(t)\right\}}{t} d t .
\end{aligned}
$$

Finally, combining (48), (50), (51) and (53) yields (25). 


\section{REFERENCES}

[1] EU 5GPP SAT5G project, Available: http://sat5g-project.eu/.

[2] The 5G Future and the Role of Satellite, iDirect White Paper, 2019, Available: https://www.idirect.net/wp-content/uploads/ 2019/01/The-5G-Future-and-the-Role-of-Satellite-White-Paper-2019.pdf, achieved at Aug. 19, 2020.

[3] C. Ge et al., "QoE-assured live streaming via satellite backhaul in 5G networks," IEEE Trans. Broadcast., vol. 65, no. 2, pp. 381-391, June 2019.

[4] A. Guidotti et al., "Architectures and key technical challenges for 5G systems incorporating satellites," IEEE Trans. Veh. Technol., vol. 68, no. 3, pp. 2624-2639, Mar. 2019.

[5] C. Niephaus, M. Kretschmer and G. Ghinea, "QoS provisioning in converged satellite and terrestrial networks: A survey of the state-of-the-art," IEEE Commun. Surveys Tuts., vol. 18, no. 4, pp. 2415-2441, Fourth quarter 2016.

[6] S. Lin, D. J. Costello and M. J. Miller, "Automatic-repeat-request error-control schemes," IEEE Commun. Mag., vol. 22, no. 12, pp. 5-17, Dec. 1984.

[7] H. Chen, R. G. Maunder and L. Hanzo, "A survey and tutorial on low-complexity turbo coding techniques and a holistic hybrid ARQ design example,” IIEEE Commun. Surveys Tuts., vol. 15, no. 4, pp. 1546-1566, Fourth Quarter 2013.

[8] A. R. Prasad, Y. Shinohara and K. Seki, "Performance of hybrid ARQ for IP packet transmission on fading channel," IEEE Trans. Veh. Tech., vol. 48, no. 3, pp. 900-910, May 1999.

[9] P. Larsson, L. K. Rasmussen and M. Skoglund, "Throughput analysis of ARQ schemes in Gaussian block fading channels," IEEE Trans. Commun., vol. 62, no. 7, pp. 2569-2588, July 2014.

[10] B. Maham, A. Behnad and M. Debbah, "Analysis of outage probability and throughput for half-duplex hybrid-ARQ relay channels," IEEE Trans. Veh. Technol., vol. 61, no. 7, pp. 3061-3070, Sept. 2012.

[11] H. Ding, S. Ma, C. Xing, Z. Fei, Y. Zhou and C. L. P. Chen, "Analysis of hybrid ARQ in Ad Hoc networks with correlated interference and feedback errors," IEEE Trans. Wireless Commun., vol. 12, no. 8, pp. 3942-3955, August 2013.

[12] A. Chelli and M. Alouini, "On the performance of hybrid-ARQ with incremental redundancy and with code combining over relay channels," IEEE Trans. Wireless Commun., vol. 12, no. 8, pp. 3860-3871, August 2013.

[13] Y. Li, M. C. Gursoy and S. Velipasalar, "On the throughput of hybrid-ARQ under statistical queuing constraints," IEEE Trans. Veh. Technol., vol. 64, no. 6, pp. 2725-2732, June 2015.

[14] P. Larsson, L. K. Rasmussen and M. Skoglund, "Throughput analysis of hybrid-ARQ matrix exponential distribution approach,” IEEE Trans. Commun., vol. 64, no. 1, pp. 416-428, Jan. 2016.

[15] A. Chelli, E. Zedini, M. Alouini, J. R. Barry and M. Pzold, "Performance and delay analysis of hybrid ARQ with incremental redundancy over double Rayleigh fading channels," IEEE Trans. Wireless Commun., vol. 13, no. 11, pp. 6245-6258, Nov. 2014.

[16] Z. Shi, H. Ding, S. Ma and K. Tam, “Analysis of HARQ-IR over time-correlated Rayleigh fading channels," IEEE Trans. Wireless Commun., vol. 14, no. 12, pp. 7096-7109, Dec. 2015.

[17] Z. Shi, S. Ma, G. Yang and M. Alouini, "Energy-efficient optimization for HARQ schemes over time-correlated fading channels," IEEE Trans. Veh. Technol., vol. 67, no. 6, pp. 4939-4953, June 2018.

[18] Z. Shi, S. Ma, G. Yang, K. Tam and M. Xia, "Asymptotic outage analysis of HARQ-IR over time-correlated Nakagami-m fading channels," IEEE Trans. Wireless Commun., vol. 16, no. 9, pp. 6119-6134, Sept. 2017.

[19] H. Jin, C. Cho, N. Song and D. K. Sung, "Optimal rate selection for persistent scheduling with HARQ in time-correlated Nakagami-m fading channels," IEEE Trans. Wireless Commun., vol. 10, no. 2, pp. 637-647, Feb. 2011. 
[20] E. Zedini, A. Chelli and M. Alouini, "On the performance analysis of hybrid ARQ With incremental redundancy and with code combining over free-space optical channels with pointing errors," IEEE Photon. J., vol. 6, no. 4, pp. 1-18, Aug. 2014.

[21] S. M. Aghajanzadeh and M. Uysal, "Information theoretic analysis of hybrid-ARQ protocols in coherent free-space optical systems," IEEE Trans. Commun., vol. 60, no. 5, pp. 1432-1442, May 2012.

[22] H. Ding, Z. Shi, S. Ma and C. Xing, "On the performance of HARQ-IR over Nakagami-m fading channels in mobile Ad Hoc networks," IEEE Trans. Veh. Technol., vol. 66, no. 5, pp. 3913-3929, May 2017.

[23] Z. Xiang, W. Yang, G. Pan, Y. Cai, Y. Song and Y. Zou, "Secure transmission in HARQ-assisted non-orthogonal multiple access networks," IEEE Trans. Inf. Forensics Secur., vol. 15, pp. 2171-2182, 2020.

[24] S. Lin and P. Yu, "A hybrid ARQ scheme with parity retransmission for error control of satellite channels," IEEE Trans. Commun., vol. 30, no. 7, pp. 1701-1719, July 1982.

[25] R. H. Deng, "Hybrid ARQ schemes for point-to-multipoint communication over nonstationary broadcast channels," IEEE Trans. Commun., vol. 41, no. 9, pp. 1379-1387, Sept. 1993.

[26] O. A. Gonzalez and R. Kohno, "A spread slotted CDMA/ALOHA system with hybrid ARQ for satellite multiple access," IEEE J. Sel. Areas Commun., vol. 18, no. 1, pp. 123-131, Jan. 2000.

[27] J. Zhu, S. Roy and J. H. Kim, "Performance modelling of TCP enhancements in terrestrial satellite hybrid networks," IEEE/ACM Trans. Netw., vol. 14, no. 4, pp. 753-766, Aug. 2006.

[28] J. Jiao, Y. Hu, Q. Zhang and S. Wu, "Performance modeling of LTP-HARQ schemes over OSTBC-MIMO channels for hybrid satellite terrestrial networks," IEEE Access, vol. 6, pp. 5256-5268, 2018.

[29] J. Ye, C. Zhang, H. Lei, G. Pan, Z. Ding, "Secure UAV-to-UAV systems with spatially random UAVs," IEEE Wireless Commun. Lett., vol. 8, no. 2, pp. 564-567, Apr. 2019.

[30] G. Pan, H. Lei, J. An, et al., "On the secrecy of UAV systems with linear trajectory," IEEE Trans. Wireless Commun., doi: 10.1109/TWC.2020.3002230.

[31] G. Pan, J. Ye and Z. Ding, "Secure hybrid VLC-RF systems with light energy harvesting," IEEE Trans. Commun., vol. 65, no. 10, pp. 4348-4359, Oct. 2017.

[32] G. Pan, J. Ye, Y. Zhang, et al., "Performance analysis and optimization of cooperative satellite-aerial-terrestrial systems," IEEE Trans. Wireless Commun., doi: 10.1109/TWC.2020.3004644.

[33] P. Wu and N. Jindal, "Performance of hybrid-ARQ in block-fading channels: A fixed outage probability analysis," IEEE Trans. Commun., vol. 58, no. 4, pp. 1129-1141, April 2010.

[34] A. Abdi, W. Lau, M.-S. Alouini, and M. Kaveh, "A new simple model for land mobile satellite channels: First and second order statistics" IEEE Trans. Wireless Commun., vol. 2, no. 3, pp. 519-528, May 2003.

[35] I.S. Gradshteyn and I.M. Ryzhik, Table of Integrals, Series and Products, 7 Ed. San Diego: Academic Press, 2007.

[36] H. Zhao, L. Yang, G. Pan, and M.-S. Alouini, "Secrecy outage analysis over fluctuating two-ray fading channels," Electron. Lett., vol. 55, no. 15, pp. 866-868, Jul. 2019.

[37] M. R. Bhatnagar and Arti M.K., "On the closed-form performance analysis of maximal ratio combining in shadowed-Rician fading LMS channels," IEEE Commun. Lett., vol. 18, no. 1, pp. 54-57, Jan. 2014.

[38] A. P. Prudnikov, Y. A. Brychkov, and O. I. Marichev, Integrals and Series: More Special Functions, vol. 3, 3rd ed., New York: Gordon \& Breach Sci. Publ., 1992.

[39] V. S. Adamchik and O. I. Marichev, "The algorithm for calculating integrals of hypergeometric type functions and its realization in REDUCE system," in Proceedings of the international symposium on Symbolic and algebraic computation (ISSAC' 90), Tokyo, Japan, Aug. 1990, pp. 212-224. 